\title{
Angiogenèse : relations avec le cancer et maladies angiogéniques
}

\begin{abstract}
Une mini-synthèse consacrée en 1988 aux facteurs angiogéniques $(\mathrm{m} / \mathrm{s}$ $n^{\circ} 5$, vol. $4, p .318$ ) concluait que le contrôle de l'angiogenèse pourrait devenir important dans deux situations pathologiques : le cancer d'une part, des maladies relevant d'un dérèglement de la vascularisation d'autre part. Ces conclusions sont étayées par deux types de travaux récents.
\end{abstract}

- Angiogenèse et cancer. Les interrelations entre ces deux processus ont pu être précisées par deux équipes utilisant des modèles très différents. Judah Folkman (Boston, MA, USA), le plus ardent promoteur du rôle de l'angiogenèse dans le cancer, a utilisé [1] des souris transgéniques pour un oncogène hybride exprimant l'oncoprotéine T du SV40 placée sous la direction des séquences régulatrices de l'insuline. Une lignée de souris transgéniques produit l'oncogène dès la naissance dans toutes les cellules $\beta$ du pancréas. Vers la dixième semaine, la moitié des ilôts de Langerhans présente un aspect hyperplasique. Or, un petit nombre d'entre eux seulement se transforme en carcinomes; ces derniers, et eux seuls, montrent tous des capillaires en développement. L'induction de l'angiogenèse semble donc constituer un événement important pour la conversion d'un épithélium normal en tissu cancéreux.

Le second modèle fait état de la découverte d'un inhibiteur de la néovascularisation, étroitement lié à la présence d'un gène inhibiteur du cancer. Il est proposé par une équipe de Chicago (USA) dirigée par N.P. Bouck [2]. Elle utilise des cellules d'une lignée de rein de bébé hamster (BHK) qui, à l'image des NIH 3T3, ne réclament qu'une étape pour devenir malignes. Ce changement est attribué à la perte d'un gène récessif suppresseur du cancer. En cérisent acquièrent un pouvoir angiogénique, démontrable in vivo dans le test de la cornée du lapin, comme in vitro dans le test de migration des cellules endothéliales. Dans le milieu de culture des cellules, on trouve normalement une activité inhibitrice de l'angiogenèse, portée par une protéine de $140 \mathrm{kDa}$ que les auteurs ont purifiée mais dont ils n'ont pas cloné le gène, et qui disparaît lors de la transformation maligne. Ces expériences sont encore renforcées par l'emploi d'un mutant thermosensible : à $32^{\circ} \mathrm{C}$ le gène suppresseur du cancer est actif et la protéine 140 est présente, à $38^{\circ} \mathrm{C}$ le gène est inactif et la protéine absente. Bien que cette protéine ne semble pas être elle-même le produit direct du gène suppresseur, sa production est sous la dépendance de ce gène. Son identification complète est en cours. Ces expériences montrent l'importance de l'angiogenèse dans des modèles privilégiés. Il reste à vérifier son rôle éventuel dans d'autres systèmes. Ce travail risque d'être d'autant plus difficile [3] que beaucoup de facteurs de croissance sont sécrétés par des cellules transformées, et que la plupart de ces facteurs, sinon tous, peuvent se montrer angiogéniques.

- Maladies de l'angiogenèse. En dehors du cancer la régulation de l'angiogenèse est stricte; celle-ci ne s'exerce qu'au cours de la réparation des tissus après blessure, et s'arrête dès que cette réparation est terminée. Dans certaines circonstances pathologiques toutefois, un stimulus puissant peut déborder les barrières physiologiques, conduisant à une symptomatologie grave, comme dans la rétinite diabétique et certains hémangiomes. Pour s'y opposer, on tente de mettre au point des inhibiteurs de l'angiogenèse, dont les plus prometteurs, dérivés de stéroïdes et de la cyclodextrine font l'objet d'une «brève » (p. 515 de ce numéro). En attendant de disposer en clinique de ces agents, il est déjà possible d'améliorer une maladie grave, comme le montre l'observation de médecins de Denver et Saint-Louis (USA) [4]. L'hémangiomatose pulmonaire est une maladie rare, caractérisée par une prolifération diffuse des microvaisseaux pulmonaires. Elle atteint enfants ou adultes jeunes, aboutissant à la mort après hypertension pulmonaire ou hémorragies. White et al. [4] décrivent l'histoire d'un garçon de 12 ans dont l'état pulmonaire s'aggravait depuis quatre ans. Un traitement par l'interféron $\alpha-2 \mathrm{a}$ à la dose de trois millions d'unités par $\mathrm{m}^{2}$ de surface corporelle pendant 14 mois l'a cliniquement normalisé, en même temps que s'atténuaient les images vasculaires pulmonaires anormales constatées à l'angiographie. Ce travail soulève donc beaucoup d'espoirs pour le traitement de tels cas, considérés auparavant comme désespérés.

J.C.D.

1. Folkman J, Watson $\mathrm{K}$, Ingber D, Hanahan $\mathrm{D}$. Induction of angiogenesis during the transition from hyperplasia to neoplasia. Nature $1989 ; 339: 58-61$.

2. Rastinejad F, Polverini PJ, Bouck NP. Regulation of the activity of a new inhibitor of angiogenesis by a cancer suppressor gene. Cell $1989 ; 56: 345-55$.

3. Ross R. Successful growth of tumors. Nature 1989 ; 339 : 16-7.

4. White CM,. Sondheimer HM, Crouch EC, Wilson H, Fan LL. Treatment of pulmonary hemangiomatosis with recombinant interferon $\alpha$-2a. N Engl J Med 1989; 320 : 1197-200 